\title{
Bilateral nerve sparing robotic-assisted radical prostatectomy is associated with faster continence recovery but not with erectile function recovery compared with retropubic open prostatectomy: The need for accurate selection of patients
}

\author{
GIUSEPPE MARIO LUDOVICO ${ }^{1}$, GIUSEPPE DACHILLE ${ }^{2}$, GIOVANNI PAGLIARULO ${ }^{1}$, \\ CAROLINA D'ELIA ${ }^{3}$, NICOLA MONDAINI ${ }^{4}$, MAURO GACCI $^{5}$, BEATRICE DETTI ${ }^{6}$, \\ GIANNI MALOSSINI ${ }^{3}$, RICCARDO BARTOLETTI ${ }^{4}$ and TOMMASO CAI ${ }^{3}$ \\ ${ }^{1}$ Department of Urology and Robotic Surgery, Miulli Hospital, Acquaviva delle Fonti; ${ }^{2}$ Department of Urology, \\ San Giacomo Hospital, Monopoli, Bari; ${ }^{3}$ Department of Urology, Santa Chiara Hospital, Trento; \\ ${ }^{4}$ Department of Urology, Santa Maria Annunziata Hospital; ${ }^{5}$ Department of Urology and \\ ${ }^{6}$ Radiotherapy Unit, Careggi Hospital, University of Florence, Florence, Italy
}

Received June 12, 2012; Accepted August 13, 2012

DOI: $10.3892 / o r .2013 .2365$

\begin{abstract}
Robotic-assisted radical prostatectomy (RARP) shows measurable advantages, compared to conventional open surgery, even if some aspects are, still, under debate. The aim of this study was to compare the potency recovery rate of patients with clinically localised prostate cancer treated by bilateral nerve-sparing (BNS) RARP or retropubic radical prostatectomy (RRP), and secondarily, the urinary continence recovery evaluation and the oncological efficacy. All patients treated with BNS-RARP or BNS-RRP for clinically localised prostate cancer, performed by a single dedicated surgeon, between January 2004 and December 2008, were enrolled in this non-randomised prospective comparative study. The International Index of Erectile Function (IIEF) and erection hardness score (EHS), in the form of a questionnaire, were self-administered to each patient pre-operatively and after 12 months. The presence of surgical margins was considered as oncological outcome measure. Eighty-two patients underwent BNS-RARP while 48 underwent BNS-RRP. For BNS-RARP and BNS-RRP the median operative time was 221 and 103 min, respectively $(\mathrm{P}<0.001 ; \mathrm{df}=128 ; \mathrm{t}=721.43)$, and intra-operative blood loss was 280 and $565 \mathrm{ml}$, respectively $(\mathrm{P}<0.001 ; \mathrm{df}=128 ; \mathrm{t}=1742.44)$. At a mean follow-up period of $12.4 \pm 2.3$ months, 12 patients $(25 \%)$ in the BNS-RRP group and $22(26.8 \%)$ in the BNS-RARP group were considered potent with or without drugs $(\mathrm{P}=0.81)$. Moreover, we did not find any
\end{abstract}

Correspondence to: Dr Tommaso Cai, Department of Urology, Santa Chiara Regional Hospital, Largo Medaglie d'Oro 9, I-38123 Trento, Italy

E-mail: ktommy@libero.it

Key words: prostatic neoplasms, laparoscopy, surgery, robotics, erectile dysfunction, urinary continence statistically significant difference between the 2 groups in terms of IEFF and EHS scores after treatment (17.21 vs. 16.98; $\mathrm{P}=0.16$ and 2.1 vs. $2.0 ; \mathrm{P}=0.54)$. On the other hand, statistically significant differences between the 2 groups were found in terms of faster urinary continence recovery and the presence of positive surgical margins $(\mathrm{P}<0.001, \mathrm{P}=0.009)$. Shorter catheterization duration (7 vs. 3 days) and post-operative hospital stays ( 8 vs. 4 days; $\mathrm{P}<0.001$ ) were found in the BNS-RARP group compared to the BNS-RRP group. In conclusion, our results demonstrate that BNS-RARP does not improve erectile function recovery compared to open radical prostatectomy; however, it significantly improves urinary continence and decreases the presence of positive surgical margins.

\section{Introduction}

Radical prostatectomy (RP) is the gold standard treatment for patients with clinically localised prostate cancer (cT1-cT2) and a life expectancy of $>10$ years (1). Patient selection and surgical technique are the major determinants of post-operative erectile function (2). The shift from open to laparoscopic surgery represented a completely new experience for surgeons, who were exposed to surgical anatomy through a different perspective and were required to learn new surgical procedures and to deal with new surgical tools (2). Robotic systems have been recently introduced in order to reduce difficulties in performing complex laparoscopic urological procedures, particularly for non-laparoscopic surgeons (3). Recent studies have demonstrated that robotic-assisted radical prostatectomy (RARP) has measurable advantages, when compared to both conventional open surgery and to conventional laparoscopic prostatectomy (4), due to the fact that RARP offers the additional advantages of x10 magnified binocular, 3-dimensional visualization, motion scaling with tremor filtration, improved ergonomics and miniature wrested, articulating surgical instruments with 7 degrees of freedom (3). A number of studies comparing RARP with other 
surgical approaches have demonstrated the improved functional results of RARP. Particularly, Ficarra et al (3) showed that RARP offers better results than RRP in terms of urinary continence and erectile function recovery, with similar positive surgical margin rates. Moreover, Menon et al (5) demonsrtated that in a large series of patients, approximately $70 \%$ had recovered erectile potency after 12 months following nerve-sparing RARP. These results may be due to the fact that RARP prevents damage to the neurovascular bundle by more precise dissection and prevents inadvertent incision, traction, or incorporation of the neurovascular bundle into the suture or clip. On the other hand, Krambeck et al (6) reported no significant difference in potency rates at 1-year follow-up. Thus, the question of whether the robotic-assisted laparoscopic technique improves the chances of recovering erectile function remains under debate. The aim of this study was to compare the potency recovery rate of a contemporary series of patients with clinically localised prostate cancer treated by bilateral nerve-sparing (BNS) RARP or retropubic radical prostatectomy (RRP), performed by a single surgeon. The secondary aim of this study was the evaluation of urinary continence recovery and the oncological efficacy evaluated as the presence of surgical margins.

\section{Materials and methods}

Study design. To compare the potency recovery rate between the 2 groups of patients, those who underwent BNS RARP or BNS-RRP, all consecutive patients who had undergone BNS RP at the same Urological Unit performed by a single dedicated surgeon, between January 2004 and December 2008 were selected for this prospective study. We compared 2 series of patients who had undergone BNS-RARP and BNS-RRP performed by a single dedicated surgeon, after a learning curve of at least 50 procedures. There is no evidence-based recommendation for how long a learning curve should be; howver, the majority of published reports on RARP suggest a minimum of 50 cases (7). Each patient was evaluated for the following parameters: age, co-morbidity (age-adjusted Charlson co-morbidity index), pre-operative total PSA, biopsy Gleason score, clinical stage according to the 2002 TNM system, perineural and endovascular invasion, pathological extension of the primary tumor according to the 2002 Tumor Node Metastasis (TNM) system (8) and presence of positive surgical margins. Moreover, the International Index of Erectile Function (IIEF) and erection hardness score (EHS), in the form of a questionnaire, were self-administered to each patient, pre-operatively and after 12 months. Follow-up data were compared and analysed. Patients with no further information available at follow-up were excluded from the study. The local research ethics committee approved the study. Written informed consent was obtained from all patients. The study was conducted in line with Good Clinical Practice guidelines and with the ethical principles laid down in the latest version of the Declaration of Helsinki.

Eligibility for the study. Inclusion criteria were as follows: patients who had undergone BNS-RARP or BNS-RRP for clinically localised prostate cancer (clinical stage $<\mathrm{cT} 2 \mathrm{~b}$ ), PSA $<10 \mathrm{ng} / \mathrm{ml}$, Gleason score $<7$, life expectancy $>10$ years, pre-operative IIEF score $>25$ in accordance with Cappelleri et al (9) and an EHS of 4 (10). Patients also had to be in a stable relationship (with the same partner for $\geq 6$ months). Exclusion criteria were the presence of other neoplastic, lower urinary tract or major concomitant diseases. Patients who had undergone previous abdominal surgery were excluded. All patients with urinary incontinence or erectile dysfunction were also excluded. Moreover, we also excluded all patients receiving phosphodiesterase type 5 (PDE5) inhibitors and/or an intracorporal injection (5 $\mu \mathrm{g} /$ week) of alprostadil (PGE 1). We only enrolled patients who had undergone BNS RP by a single dedicated surgeon.

Surgical technique procedures and follow-up schedule. In brief, RRP was performed as described by Walsh (11) in 1998. RARP was performed as follows: all cases were approached transperitoneally, dissecting the anterolateral surface of the prostate using an intrafascial technique and developing the plane between the prostatic capsula and the thin surrounding periprostatic fascia. The lateral pedicles were controlled making every attempt to preserve the neurovascular bundles. Vesicourethral anastomosis was performed as described by Van Velthoven et al (12). In accordance with the European Association of Urology (EAU) guidelines, no patient had undergone lymphadenectomy due to of having lymphnode metastasis (1). It was suggested that all patients undergo an early penile rehabilitation with PDE5 inhibitors twice a week for 6 months after RP. All patients with positive margins at histopathological evaluation were included in the standard follow-up controls, but were excluded from the functional outcome analysis. Biochemical failure was defined as 2 or more consecutive ultrasensitive PSA values of $>0.2 \mathrm{ng} / \mathrm{ml}$ (1). All biochemical failure patients underwent external beam radiotherapy and/or hormonal therapy, as suggested in the EAU guidelines (1).

Questionnaires and clinical evaluation. We used the IIEF questionnaire as it has a higher level of validity, accuracy and reliability, and is more stable than single-item assessment. In accordance with Briganti et al (13), recovery of erectile function following BNS-RP was defined as an IIEF domain score of $\geq 22$. We used the EHS due to the fact that it is a simple, practical tool for clinical use (14). None of the patients received neoadjuvant or adjuvant hormonal therapy and none of the patients underwent adjuvant radiation therapy. Continent patients were defined by use of 0 or 1 safety pad/day. Outcome measures were erectile function recovery, urinary function recovery and the percentage of positive surgical margins. Moreover, the Clavien-Dindo classification of post-operative complications was used (15).

Pathological staging. All specimens, including prostate and seminal vesicles were examined microscopically after routine preparation. The prostate was weighed and cut as whole-mount 4-mm sections. All specimens were scored according to the Gleason grading system. Microscopic extension of malignant cells to the inked surface of the resected specimen was interpreted as a positive surgical margin. The pathological stages were assigned according to the 2002 TNM classification (8).

Statistical analysis. As a null hypothesis we assumed that there were no differences between the 2 groups in terms of potency 
Table I. Clinical and pathological characteristics of all patients at the time of surgery.

\begin{tabular}{|c|c|c|c|c|}
\hline \multirow[b]{2}{*}{ Characteristics } & \multirow[b]{2}{*}{ Total } & \multicolumn{2}{|c|}{ Radical prostatectomy } & \multirow[b]{2}{*}{ P-value } \\
\hline & & BNS-RRP & BNS-RARP & \\
\hline Patients enrolled & 130 & 48 & 82 & - \\
\hline Mean age & $68.1 \pm 8.7$ & $66.7 \pm 9.9$ & $68.1 \pm 2.3$ & 0.22 \\
\hline Educational level & & & & 0.004 \\
\hline Primary school & 55 & 25 & 30 & \\
\hline High school & 34 & 15 & 19 & \\
\hline Degree & 41 & 8 & 33 & \\
\hline Pre-operative tPSA & $8.2 \pm 1.1$ & $7.9 \pm 2.1$ & $8.3 \pm 3.1$ & 0.38 \\
\hline Biopsy Gleason score & & & & 0.15 \\
\hline $6(3+3)$ & 67 & 29 & 38 & \\
\hline $7(3+4)$ & 41 & 12 & 29 & \\
\hline $7(4+3)$ & 22 & 7 & 15 & \\
\hline Clinical stage & & & & 0.59 \\
\hline cT1 & 56 & 31 & 25 & \\
\hline cT2a & 74 & 37 & 37 & \\
\hline Charlson CI & $6.1 \pm 1.2$ & $6.3 \pm 1.1$ & $6.0 \pm 0.8$ & 0.07 \\
\hline Pre-operative IIEF & $28.3 \pm 1.1$ & $28.9 \pm 0.9$ & $29.1 \pm 1.3$ & 0.34 \\
\hline Pre-operative EHS & 4 & 4 & 4 & - \\
\hline
\end{tabular}

recovery rate, urinary continence recovery and the presence of surgical margins. The comparison of continuous variables between the 2 groups was performed with the Student's t-test, Mann-Whitney U test or Wilcoxon test, where appropriate. Time-related changes of continuous variables were analysed using the paired t-test. Pearson's Chi-square and Fisher's exact test were used to compare categorical variables and proportions, where appropriate. A two-tailed probability P-value of $<0.05$ was considered to indicate a statistically significant difference. All statistical calculations were performed with the Statistical Package for Social Sciences v.11.0 (SPSS, Inc., Chicago, IL, USA). Statistical tests were carried out with a two-sided $\alpha$ level of 0.05 .

\section{Results}

Among 400 consecutive patients undergoing RP during the wide study period, 135 patients were eventually eligible for the present study. This trial ultimately included 130 patients. Eighty-two received BNS-RARP and 48 BNS-RRP. Patient characteristics, clinical and laboratory data at enrollment are described in Table I, according to the group. At the baseline, all patients were pre-operatively continent and potent. The 2 groups are statistically comparable. For BNS-RARP and BNS-RRP the median operative time was 221 and $103 \mathrm{~min}$, respectively $(\mathrm{P}<0.001 ; \mathrm{df}=128 ; \mathrm{t}=721.43)$ and intra-operative blood loss was 280 and $565 \mathrm{ml}$, respectively $(\mathrm{P}<0.001$; $\mathrm{df}=128$; $\mathrm{t}=1742.44)$. All perioperative data and complications are described in Table II.

Functional outcome evaluation. All patients correctly underwent penile rehabilitation with oral PDE5 inhibitors twice in a week for 6 months after surgical treatment. No patient discontinued the treatment. All data are summarised in Table III.

Erectile function. In patients undergoing BNS-RRP we documented the following post-operative results: return to baseline of the erectile function (IIEF score $>25$ and EHS score 4) was present in 3 patients $(6 \%)$, with a mean IIEF score of 28.5 (range 27-30) and pharmacological-induced potency (IIEF score $>25$ and EHS score 4) was present in 9 patients (19\%), with a mean IIEF score of 26.3 (range 25-30), while we registered the absence of recovery of the erectile function in 36 patients $(75 \%)$. Then, 12 patients $(25 \%)$ were considered potent, with a mean IIEF score of 27.2 (range 25-30). On the other hand, in patients undergoing BNS-RARP, the data showed the following post-operative results: return to baseline of the erectile function (IIEF score $>25$ and EHS score was 4) was present in 3 patients (4\%), with a mean IIEF score of 27.9 (range 27-30) and pharmacological-induced potency (IIEF score $>25$ and EHS score 4 ) in 19 patients (23\%), with a mean IIEF score of 26.7 (range 25-30), while we registered the 
Table II. Perioperative data and complications.

\begin{tabular}{|c|c|c|c|}
\hline & \multicolumn{2}{|c|}{ Radical prostatectomy } & \multirow[b]{2}{*}{ P-value } \\
\hline & BNS-RRP & BNS-RARP & \\
\hline Median operative time & 221 & 103 & $<0.001$ \\
\hline Intra-operative blood loss & 280 & 565 & $<0.001$ \\
\hline Pathologic stage & & & - \\
\hline $\mathrm{pT} 2 \mathrm{a}$ & 11 & 22 & \\
\hline $\mathrm{pT} 2 \mathrm{~b}$ & 8 & 13 & \\
\hline $\mathrm{pT} 2 \mathrm{c}$ & 12 & 19 & \\
\hline pT3a & 12 & 21 & \\
\hline pT3b & 5 & 9 & \\
\hline Gleason score & & & - \\
\hline $6(3+3)$ & 23 & 38 & \\
\hline $7(3+4)$ & 18 & 27 & \\
\hline $7(4+3)$ & 7 & 17 & \\
\hline Margin status positive & 14 & 8 & 0.009 \\
\hline Clavien-Dindo (patients) & & & - \\
\hline No complications & 43 & 74 & \\
\hline Grade I-II & 4 & 7 & \\
\hline Grade III & 1 & 1 & \\
\hline Median catheterization time & 7 & 3 & $<0.001$ \\
\hline Median hospitalization time & 8 & 4 & $<0.001$ \\
\hline
\end{tabular}

BNS, bilateral nerve-sparing; RRP, retropubic radical prostatectomy; RARP, robotic-assisted radical prostatectomy.

Table III. Functional outcome evaluation.

\begin{tabular}{lcc}
\hline & \multicolumn{2}{c}{ Radical prostatectomy } \\
\cline { 2 - 3 } & BNS-RRP & BNS-RARP \\
\hline Erectile function & & 16.98 \\
Total IIEF mean & 17.21 & 0.54 \\
Total EHS & 2.12 .0 & $60(73.1)$ \\
Absence of recovery, $(\%)$ & $36(75)$ & $19(23.2)$ \\
Return to baseline (with drugs), $\mathrm{n}(\%)$ & $9(18.7)$ & 26.7 \\
IIEF mean score & 26.3 & 4 \\
EHS median & 4 & 27.9 \\
Return to baseline (without drugs), $\mathrm{n}(\%)$ & $3(6.3)$ & 4 \\
IIEF mean score & 28.5 & - \\
EHS median & 4 & $78(95.1)$ \\
Urinary function & & $4.1 \pm 0.73$ \\
Continent patients & $47(97.9)$ & - \\
Continence recovery time (months) & $6.9 \pm 1.4$ & 0.74 \\
\hline
\end{tabular}

BNS, bilateral nerve-sparing; RRP, retropubic radical prostatectomy; RARP, robotic-assisted radical prostatectomy; IIEF, International Index of Erectile Function; EHS, erection hardness score. 
absence of recovery of erectile function in 60 patients $(73 \%)$. Then, 22 patients $(26.8 \%)$ were considered potent, with a mean IIEF score of 27.1 (range 25-30). We did not find statistically significant differences in erectile function recovery achieved after BNS-RARP, when compared with BSN-RRP. In fact, we did not find statistically significant differences between the 2 wide groups in terms of IEFF and EHS scores after treatment (17.21 vs. $16.98 ; \mathrm{P}=0.16$ and 2.1 vs. $2.0 ; \mathrm{P}=0.54$ ).

Urinary function. Twelve months after the procedures, 47 patients who had undergone BNS RRP (98\%) and 78 patients who had undergone BNS-RARP $(95 \%)$ were continent $(\mathrm{P}=0.74$; Chi-square, $0.10 ; \mathrm{df}=1)$. However, we found that patients who had undergone BNS-RARP had a much more rapid continence recovery than those who had undergone BNS-RRP $(4.1 \pm 0.73$ vs. $6.9 \pm 1.4$ months, respectively $(\mathrm{P}<0.001$; $\mathrm{df}=128$; $\mathrm{t}=14.9$ ).

Pathological and oncological outcome. At pathological evaluation, in the BNS-RRP group we documented the following findings: 31 pT2 (64.5\%), 17 pT3 (35.5\%); Gleason score: $236(47.9 \%), 187(3+4)(37.5 \%)$ and $7(4+3)(14.6 \%)$. On the other hand, in the BNS-RARP group: 52 pT2 (63.4\%), 30 pT3 (36.6\%); Gleason score: 386 (46.3\%), $277(3+4)(32.9 \%)$ and $17(4+3)(20.8 \%)$. Overall positive surgical margins were found in 14 patients who had undergone BNS-RRP (30\%) and in 8 patients who had undergone BNS-RARP (10\%) $(\mathrm{P}=0.009 ; \mathrm{df}=1$; Chi-square, 6.79); moreover, positive surgical margins in patients with localised prostate cancer were found in 3 patients who had undergone BNS-RRP and in 2 patients who had undergone BNS-RARP.

Complications and post-operative characteristics. We documented overall complication rate of $10.4 \%$ in the BNS-RRP group (5 patients) and of $9.7 \%$ in the BNR-RARP group (8 patients). Specifically, we registered 6 low grade complications (Clavien I and II) in 4 patients in the retropubic serie and 9 low grade complications in 7 patients in the robotic serie. Data showed, moreover, 2 high grade complications in 1 patient in the retropubic and 1 in the robotic group. We did not show Clavien V complication (death) in both group. Patients of the BNS-RRP group removed the catheter in 7 post-operative day and were discharged in 8 post-operative day, while patients of the BNS-RARP group removed the catheter in 3 post-operative day and were discharged in 4 post-operative day, respectively $(\mathrm{P}<0.001 ; \mathrm{df}=128 ; \mathrm{t}=24.4)$.

\section{Discussion}

Currently, the main challenge in the treatment of prostate cancer patients is the improvement of quality of life, due to the fact that oncological outcomes have improved (6). The use of robotic-assisted laparoscopic radical prostatectomy has been purposed in order to obtain better results in terms of quality of life and oncological outcomes (16). However, the question of whether the robotic-assisted laparoscopic technique improves the chances of recovering from urinary continence and recovering erectile function remains under debate. The cost effectiveness of the new technique remains an issue. RARP is associated with a higher cost and a much longer learning curve than radical open prostatectomy and radical laparoscopic prostatectomy (3). In our series of patients, we did not find any statistically significant difference between the 2 groups in terms of erectile function recovery when compared to open RP. However, compared to open RP, RARP significantly improved continence recovery and decreased the presence of positive surgical margins. Our findings differ from those reported by other authors. Rocco et al (17) reported higher potency rates after RARP than open RP at 3, 6 and 12 months (RARP 31, 43 and $61 \%$, respectively; open RP 18,31 and $41 \%$, respectively; $\mathrm{P}=0.006,0,045$ and 0.003 , respectively). Moreover, Tewari et al (18) compared 110 patients who had undergone RRP with 200 patients who had undergone RARP. In this study, only patients who had BNS-RRP or BNS-RARP and were potent pre-operatively were included. Following RARP, the patients had a more rapid erectile function recovery, with $50 \%$ at a mean follow-up of 180 vs. 440 days after RRP $(\mathrm{P}<0.05)(18)$.

Clearly, it is well known that RARP has greater incidence of neurovascular bundle preservation and faster convalescence than RRP, but it is also well known, that the success of the nerve-sparing technique is determined by the accurate selection of patients (19-20). As suggested by Novara et al (21), in the era of robotic surgery, the key point for the success of the nerve-sparing technique is the accurate selection of patients. At the age of $<65$ years, the absence of associated co-morbidities and good pre-operative erectile function are the most important pre-operative factors to select those patients for whom BNS-RARP can achieve the best results (21). Even though we enrolled patients with a pre-operative IIEF score of $>25$, an EHS of 4 and who were in a stable relationship for $\geq 6$ months, we registered the absence of recovery of erectile function in 36 patients $(75 \%)$ in the BNS-RRP group and in 60 patients $(73 \%)$ in the BNS-RARP group. The reason for this low erectile recovery rate may be that in our series of patients, the main parameter affecting erectile function recovery was an age of $>65$ years. Ninety-eight patients $(75.3 \%)$, were $>65$ years old and this is possibly the reason for our results. However, in everyday clinical practice, most patients are $>65$ years old. The small number of enrolled patients, however, did not allow us to perform a multivariate analysis in order to establish the independent factors affecting erectile recovery. In this sense, the relatively small number of patients was the first main limitation of our study. On the other hand, we observed a good continence recovery rate in both groups of patients, demonstrating that age is not a factor affecting continence recovery probability. Another limitation of this study was the short-term follow-up period. However, several studies have stated that urinary and sexual function recovery can be evaluated within 12 months and that the probability of recovery after this time is very low (6). However, the stronger point of this study was the fact that all patients had undergone RP by a single dedicated surgeon after a learning curve of at least 50 patients. Finally, we found a higher frequency of positive surgical margins in the BNS-RRP group compared with the BNS-RARP group. These data, however, are in line with the literature (3).

In conclusion, we noted that, in our series of patients, BNS-RARP did not improve erectile recovery when compared to open RP, but allowed for a more rapid recovery from urinary continence and improved the oncological outcome in 
terms of lower positive surgical margins prevalence. However, prospective studies with longer follow-up periods and a greater number of patients are required to compare the oncological and functional results.

\section{Acknowledgements}

We are grateful to Professor John Denton for the manuscript language revision.

\section{References}

1. Heidenreich A, Bellmunt J, Bolla M, Joniau S, Mason M Matveev V, Mottet N, Schmid HP, van der Kwast T, Wiegel T and Zattoni F; European Association of Urology: EAU guidelines on prostate cancer. Part 1: screening, diagnosis, and treatment of clinically localised disease. Eur Urol 59: 61-71, 2011.

2. Magheli A and Burnett AL: Erectile dysfunction following prostatectomy: prevention and treatment. Nat Rev Urol 6: 415-427, 2009.

3. Ficarra V, Novarra G, Artibani W, Cestari A, Galfano A, Graefen M, Guazzoni G,Guillonneau B, Menon M, Montorsi F, et al: Retropubic, laparoscopic, and robot-assisted radical prostatectomy: a systematic review and cumulative analysis of comparative studies. Eur Urol 55: 1037-1063, 2009.

4. Ou YC, Yang CR, Wang J, Cheng CL and Patel VR: Comparison of robotic-assisted versus retropubic radical prostatectomy performed by a single surgeon. Anticancer Res 29: 1637-1642, 2009.

5. Menon M, Muhletaler F, Campos M and Peabody JO: Assesment of early continence after reconstruction of the periprostatic tissues in patients undergoing computer assisted (robotic) prostatectomy: results of a 2 group parallel randomized controlled trial. J Urol 180: 1018-1023, 2008.

6. Krambeck AE, DiMarco DS and Rangel LJ: Radical prostatectomy for prostatic adenocarcinoma: a matched comparision of open retropubic and robot-assisted techniques. BUJ Int 103: 488-453, 2009

7. Jaffe J, Castellucci S, Cathelineau X, Harmon J, Rozet F, Barret E and Vallancien G: Robot-assisted laparoscopic prostatectomy: a single institution's learning curve. Urology 73: 127-133, 2009.

8. Greene FL, Page DL, Fleming ID, et al: AJCC Cancer Staging Manual. 6th edition. Springer-Verlag, New York, 2002.

9. Cappelleri JC, Rosen RC, Smith MD, Mishra A and Osterloh IH: Diagnostic evaluation of the erectile function domain of the International Index of Erectile Function. Urology 54: 346-351, 2009.
10. Mulhall JP, Goldstein I, Bushmakin AG, Cappelleri JC and Hvidsten K: Validation of the erection hardness score. J Sex Med 4: 1626-1634, 2007.

11. Walsh PC: Anatomic radical prostatectomy: evolution of the surgical technique. J Urol 160: 2418-2424, 1998.

12. Van Velthoven RF, Ahlering TE, Peltier A, Skarecky DW and Clayman RV: Technique for laparoscopic running urethrovesical anastomosis: the single knot method. Urology 61: 699-702, 2003.

13. Briganti A, Di Trapani E, Abdollah F, Gallina A, Suardi N, Capitanio U, Tutolo M, Passoni N, Salonia A, Digirolamo V, et al: Choosing the best candidates for penile rehabilitation after bilateral nerve-sparing radical prostatectomy. J Sex Med 9: 608-617, 2012.

14. Hwang TI, Tsai TF, Lin YC, Chiang HS and Chang LS: A survey of erectile dysfunction in Taiwan: use of the erection hardness score and quality of erection questionnaire. J Sex Med 7: 2817-2824, 2010

15. Dindo D, Demartines N and Clavien PA: Classification of surgical complications: a new proposal with evaluation in a cohort of 6336 patients and results of a survey. Ann Surg 240: 205-213, 2004.

16. Masterson TA, Cheng L, Boris RS and Koch MO: Open vs. robotic-assisted radical prostatectomy: A single surgeon and pathologist comparison of pathologic and oncologic outcomes. Urol Oncol: Jan 3, 2012 (Epub ahead of print).

17. Rocco B, Matei DV, Melegari S, Ospina JC, Mazzoleni F, Errico G, Mastropasqua M, Santoro L, Detti S and de Cobelli O: Robotic vs. open prostatectomy in a laparoscopically naive centre: a matched-pair analysis. BJU Int 104: 991-995, 2009.

18. Tewari K, Jhaveri J, Rao S, Yadav R, Bartsch G, Te A, Ioffe E, Pineda M, Mudaliar S, Nguyen L, et al: Total reconstruction of the vesico-urethral junction. BJU Int 101: 871-877, 2008.

19. Drouin SJ, Vaessen C, Misraï V, Ferhi K, Bitker MO, ChartierKastler E, Haertig A, Richard F and Rouprêt M: Oncologic and functional outcomes after robot-assisted laparoscopic radical prostatectomy. Prog Urol 19: 158-164, 2009.

20. Tsao AK, Smaldone MD, Averch TD and Jackman SV: Robot-assisted laparoscopic prostatectomy: the first 100 patients - improving patient safety and outcomes. J Endourol 23: 481-484, 2009.

21. Novara G, Ficarra V, D'Elia C, Secco S, De Gobbi A, Cavalleri S and Artibani W: Preoperative criteria to select patients for bilateral nerve-sparing robotic-assisted radical prostatectomy. J Sex Med 7: 839-845, 2010. 